\title{
Effects of Astaxanthin in patients with memory impairment
}

\author{
Alexandra Cristina Butunoi, Cristina-Maria Gavrilescu, \\ Paloma Lascarache, Elena Cojocaru, Roxana Mihaela Barbu
}

\begin{abstract}
Alexandra Cristina Butunoi - Specialist in Geriatrics - Gerontology, UMS Răducăneni, Romania. Cristina-Maria Gavrilescu - Lecturer, MD, PhD, „Grigore T. Popa” University of Medicine and Pharmacy, Iași, Ist Medical Department, Senior in Internal Medicine, Specialist in Geriatrics, specialist în Clinical Pharmacology, Romania.
\end{abstract}

Paloma Lascarache - Lecturer, MD, PhD, „Grigore T. Popa” University of Medicine and Pharmacy, Iași, Ist Medical Department, Senior in Internal Medicine, Senior in Cardiology, Romania.

Elena Cojocaru - Lecturer, MD, PhD, Department of Physiology „Grigore T. Popa” University of Medicine and Pharmacy, Iași, Senior in Laboratory Medicine, Romania.

Roxana Mihaela Barbu - MD, PhD, Department of Physiology, University of Medicine and Pharmacy „Grigore T. Popa”, Iași, Specialist in Clinical Pharmacology, Romania.

\begin{abstract}
Introduction: Astaxanthin is used to treat a variety of cardiovascular, metabolic, ophthalmological, immune, degenerative, and psychiatric disorders. We aimed to perform a systematic review on the effects of astaxanthin in people with mental disorders. Material and method: Several medical databases were searched: PubMed, Embase, Cochrane, PMC to identify randomized studies for both humans and animals on the effects of this antioxidant on cognitive function. Results and discussions: Analyzed studies show that Astaxanthin is a carotenoid with strong antioxidant properties. It is useful as a supplement for the positive effects on the health of the cardiovascular system, immunity, but also on mental health. Due to the fact that Astaxanthin can cross the blood-brain barrier, it plays a very important role in everything related to the activity of the brain, protecting the nervous system from the harmful effects of the oxidative stress. Astaxanthin has direct effects on memory disorders present in young people as well as in the elderly, improving memory and ability to concentrate. Conclusions: This review of the data showed that astaxanthin has direct effects on the risk factors that trigger memory disorders. It reduces the risk of developing cardiovascular disease, but also protects directly and indirectly the activity of the brain, diminishing the harmfull effects of the oxidative stress on the nervous system. It is a very useful natural supplement for memory disorders, helping mental health by improving
\end{abstract}


s cognitive function. Further studies are needed to better understand the effects of astaxanthin on all human memory disorders, but it has been shown to improve memory and the ability to concentrate. Astaxanthin could be useful in treating Alzheimer's disease, Parkinson and for reducing the consquencies of stroke.

\section{KEYWORDS:}

\section{Astaxanthin; beneficial effects, memory disorders.}

\section{INTRODUCTION}

Memory is defined as the brain's ability to retain and remember various information, experiences, procedures, abilities, and habits. There are factors that influence the normal activity of the brain, triggering various memory problems from senility, dementia or Alzheimer. Cognitive disorders are characterized by changes in brain structure and function that affect learning, orientation, judgment, memory and intellectual abilities. The normal functioning of the brain can be affected by various conditions or situations, which can lead to memory degradation, subsequently appearing memory disorders. Diseases that affect memory by damaging brain cells are: Alzheimer's, stroke, head trauma and other neurodegenerative conditions (1).

Alzheimer's disease is a progressive neurodegenerative disease, a type of progressive dementia that, correlated with the aging process, is characterized by memory loss, thinking disorders, speech, behavior and personality changes, affecting millions of people globally. It is known to be the most common cause of dementia in the elderly, causing major progressive deficits in cognitive function and memory. Currently, over 35 million people worldwide are affected by this disease. Memory problems are not just caused by injuries or disorders that occur in the brain. Environmental, psychological, and emotional factors can have a negative impact on memory, including storage, retention, activation, subsequent retrieval, and the use of information or memories (2).

Regarding the factors that may contribute to the development of memory disorders, the literature indicates the following: genetics, aging, diet high in sugar or fat, sedentary lifestyle, disturbed chronobiology / sleep and excessive alcohol consumption. Pathogeneis is connected to reactive oxygen species, which play an important role in vascular biology and are harmful in high concentrations, aggravating stress and causing damage to all cellular components, leading thus to accelerated cellular aging $(2,3)$. Antioxidant defense decreases with age, so that we lose the ability to produce the high levels of antioxidants we need to cope with growing harmful environmental factors such as pollution, food processed, contaminants and stressors. In addition, with age, inflammation of the brain increases. The body's response is to release inflammatory compounds to aid in healing; but they can also destroy healthy cells - leading to irritation and tissue damage. Although memory loss can be an integral part of an aging brain, it is possible to slow the decline of mental abilities and maintain complete brain function in optimal conditions. The risk of developing neurodegenerative diseases, such as Alzheimer's disease, Parkinson's disease or dementia, continues to increase alarmingly with age. These conditions are characterized by the progressive loss of neurons in the brain and can lead to memory 


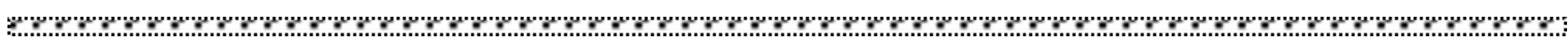

loss, confusion, tremor, agitation and anxiety.

Numerous studies have shown that some supplements, such as astaxanthin, have benefits for the brain, helping to maintain cognitive function. In a study in mice, astaxanthin supplements increased the formation of new cells in the brain and improved the spatial memory of mice $(4,5$ ,6).

Astaxanthin $\quad\left(3,3^{\prime}\right.$-dihydroxy- $\beta$-carotene-4,4'dione, AXT) is a xanthophyllic carotenoid commonly found in seafood and plants. Astaxanthin is found in the free state and in the form of an ester and also exists as a chromoprotein. Oxygen groups make ketocarotenoids relatively more polar, allow esterification and can lead to higher antioxidant activity. Astaxanthin is found in many organisms through food intake, but the primary producers of this carotenoid are limited; a number of microorganisms and plants, for example, bacteria, algae, fungi and members of the genus Adonis in higher plants. To date, green algae Haematococcus pluvialis (Chlorophyta, Volvocales) has the highest reported level of astaxanthin at $4 \%$ dry weight (7).

The main effects and benefits of Astaxanthin on the body are: decrease of reactive oxygen species, thus leading to protection of cells and cellular components against oxidative stress; decrease of $\mathrm{TNF} \alpha$, prostaglandin $\mathrm{E} 2$, interleukin $\mathrm{IL}-1 \mathrm{~b}$; modulation of cyclooxygenase COX2 and COX1; antiinflammatory effect; stimulation of antibodies production and strengthening of the immune system; protection of the brain, support to cognitive function and memory health; prevention of premature aging; reduction of pain and swelling at the osteoarticular, renal and gastrointestinal level; protection of the ocular system, improving capillary blood flow, acuity and visual accommodation at a distance, reducing eye fatigue and loss of ability to focus nearby objects, protection of retinal photoreceptors against UV radiation aggression; increase of the metabolic, mitochondrial and cardiovascular health by reducing LDL peroxidation in the vascular wall; vasodilation; improve of skin elasticity and hydration, maintaining tone, reducing collagen degradation and wrinkle formation; support for the joint health by increasing physical endurance, decrease of joint and muscle pain after intense physical exertion by reducing the level of lactic acid caused by muscle overload $(8,9,10)$.

\section{MATERIALS AND METHOD}

Several medical databases were searched: PubMed, Embase, Cochrane, PMC to identify randomized studies for both humans and animals on the effects of Astaxanthin on cognitive function.

\section{RESULTS AND DISCUSSION}

The articles were analyzed in order to find detailed analysis of the effects of astaxanthin supplementation in patients with memory disorders and provide an in-depth summary of the results of clinical trials addressing this issue primarily or indirectly. Astaxanthin is a relatively new antioxidant that has documented benefits for the human body. Almost unknown until recently, astaxanthin is a carotenoid with strong antioxidant properties. Extracted from a single-celled microalgae, it can be taken as a supplement with positive effects on the health of the cardiovascular system, on the eye system but also on mental health $(8,9)$. Astaxanthin has been shown to provide strong antioxidant protection and benefits for the whole body. Each type of antioxidant acts predominantly in certain organs having different functions of annihilation of oxidizing species specific to that organ. Astaxanthin mainly acts on small blood vessels, with positive effects on the 
heart, brain, nervous system and eyes. Perhaps the most important antioxidant action of Astaxanthin is on the mitochondria, the engine of the cell. Compared to other antioxidants, Astaxanthin has the greatest ability to annihilate the free radicals with "singular oxygen". Because Astaxanthin can cross the blood-brain barrier, it plays a very important role in everything related to the activity of the brain, protecting the nervous system of the brain from the harmful effects of oxidative stress. Therefore, Astaxanthin is useful in treating Alzheimer's disease, Parkinson's disease, or reducing the sequelae caused by a stroke. (11, 12, 13) Because Astaxanthin easily reaches the small capillaries that supply the eye, it bring its benefits and also associate synergistically with lutein and zeaxanthin (14).

Clinical studies that have measured the effects of astaxanthin proved that supplements with this substance can reduce both markers of inflammation in the body and markers of oxidative stress. Another analysis suggested that astaxanthin could protect against atherosclerosis, a condition characterized by the accumulation of fat and cholesterol in the arteries, leading to loss of elasticity (3, 9, 10). When it comes to antioxidant coverage, Astaxanthin is in a class of its own because it reaches every cell in the body. Its unique lipophilic and hydrophilic molecular properties allow it to penetrate the entire cell body, with one part of the astaxanthin molecule protecting the fat-soluble part of the cell and the other part protecting the water- soluble part of the cell. This phenomenon could be described as "an embrace of the whole cell" (11). Astaxanthin has also significant positive effects in cases of metabolic syndrome. Thus, in double-blind, randomized controlled trials, astaxanthin reduced oxidative stress in overweight and obese subjects, diabetics and smokers $(3,10$, 12). It was proved that astaxanthin increases the level of adiponectin in the blood. Adiponectin is a hormone produced by adipose tissue, heart tissue, skeletal muscle and endothelium of blood vessels. Serum adiponectin levels tend to be significantly reduced in obese, diabetic, smoking subjects, in patients with coronary heart disease, and in patients with metabolic syndrome $(12,15)$.

Recent data show that patients with metabolic syndrome have a much higher likeliness to develop Alzheimer dementia compared to those without metabolic syndrome (16). The association between metabolic syndrome and dementia brings another argument for the positive role of astaxanthine for preventing dementia in patients with metabolic syndrome. Although some symptoms of dementia cannot be controlled, there are still strategies to improve memory capacity. Changes in diet, rest schedule, physical activity and routine are simple strategies for preventing and improving memory disorders. Remedies such as Astaxanthin help prevent memory problems, maintaining the harmony and systemic balance of the brain and nervous system, without side effects or sedation (12, 13). 


\section{CONCLUSIONS}

This review of the actual state of knowledge showed that astaxanthin has direct effects on the risk factors that trigger memory disorders. It reduces the risk of developing cardiovascular disease, but also protects directly and indirectly the activity of the brain, diminishing the harmfull effects of the oxidative stress on the nervous system. It is a very useful natural supplement for memory disorders, helping mental health by improving cognitive function. Further studies are needed to better understand the effects of astaxanthin on all human memory disorders, but it has been shown to improve memory and the ability to concentrate.

The proven properties of this substance are really numerous and remarkable, occuring from its main action as an antioxidant, slowing down the degenerative processes due to aging. Astaxanthin could be useful in treating Alzheimer's disease, Parkinson, or for reducing the sequelae caused by stroke.

\section{ACKNOWLEDGEMENTS AND DISCLOSURES}

The authors declare that they have no potential conflicts of interest to disclose.

\section{REFERENCES}

1. 1. Deluca, H.F., Overview of general physiologic features and functions of vitamin D, Am. J. Clin. Nutr., 80(6), 2004, Mendiola-Precoma, J., Berumen, L. C.,K. Padilla, Garcia-Alcocer, G. Therapies for Prevention and Treatment of Alzheimer's Disease, BioMed Research International, 2016;2016:17.

2. Jessica J. Jalbert, Lori A. Daiello, Kate L. Lapane. Dementia of the Alzheimer Type. Epidemiol Rev. 2008;30(1);15-34.

3. Martins Gregório, Bianca; Benchimol De Souza, Diogo; Amorim de Morais Nascimento, Fernanda; Matta, Leonardo; Fernandes-Santos, Caroline, The Potential Role of Antioxidants in Metabolic Syndrome. Current pharmaceutical design, 2016;22(7); 859-869.

4. Jang Soo Yook, Masahiro Okamoto, Randeep Rakwal, Junko Shibato, Min Chul Lee, Takashi Matsui, Hyukki Chang, Joon Yong Cho, Hideaki Soya, Astaxanthin supplementation enhances adult hippocampal neurogenesis and spatial memory in mice. Molecular Nutrition \& Food Research, 2016;60:589-599.

5. Tang-Bin Zou, Shan-Shan Zhu, Fei Luo, Wei-Qiao Li, Xue-Rong Sun, Hong-Fu Wu. Effects of Astaxanthin on Reverse Cholesterol Transport and Atherosclerosis in Mice. BioMed Research International, 2017;2017:6.

6. Yoshimi Nakajima, Yuta Inokuchi, Masamitsu Shimazawa, Kazumasa Otsubo, Takashi Ishibashi, $\underline{\text { Hideaki Hara. }}$ Astaxanthin, a dietary carotenoid, protects retinal cells against oxidative stress in-vitro and in mice in-vivo,The Journal of Pharmacy and Pharmacology, 2008;60(10):1365-1374.

7. Thomas O. Butler, Gordon J. McDougall , Raymond Campbell , Michele S. Stanley John G. Day 2 Media Screening for Obtaining Haematococcus pluvialis Red Motile Macrozooids Rich in Astaxanthin and Fatty Acids. Biology. 2018;7(1): 2 .

8. Robert G. Fassett, Jeff S. Coombes "Astaxanthin: A Potential Therapeutic Agent in Cardiovascular Disease," Marine Drugs, 2011;9(3):447-465.

9. Adam Lauver, Samuel F. Lockwood, Benedict R. Lucchesi. Disodium Disuccinate Astaxanthin (Cardax) attenuates complement activation and reduces myocardial injury following ischemia / reperfusion.", The Journal of Pharmacology and Experimental Therapeutics, 2005;314 (2): 686-692.

10. Robert G. Fassett, Jeff S. Coombes. Astaxanthin, oxidative stress, inflammation and cardiovascular disease, Future Cardiology, 2009;213(14):456-457.

11. Qinyong Ye, Bixia Huang, Xiaodong Zhang et al. Astaxanthin protects against MPP+- induced oxidative stress in PC12 cells via the HO-1/NOX2 axis. BMC Neurosci, 2012;13(156);231-232.

12. G. Verdile, K. N. Keane, V. F. Cruzat et al. Inflammation and oxidative stress: the molecular connectivity between insulin resistance, obesity, and Alzheimer's disease. Mediators of Inflammation, 2015;5(4):321-324.

13. JL Etnier, WB Karper, JD Labban, AT Piepmeier, CH Shih, WN Dudley et al.The Physical Activity and Alzheimer's Disease (PAAD) Study: Cognitive outcomes, Annals of Behavioral Medicine. 2018;52(2):175-185.

14. Keiko Kono, Yoshiki Shimizu, Satomi Takahashi, Sayuri Matsuoka, Kei Yuib. Study "Effect of Multiple Dietary Supplement Containing Lutein, Astaxanthin, Cyanidin-3-Glucoside, and DHA on Accommodative Ability", Current Medicinal Chemistry, 2014;34(4):10-14.

15. Izuka A. et all. Astaxanthin Enhances ATP-Binding Cassette Transporter A1/G1 Expressions and Cholesterol Efflux from Macrophages. Journal of Nutritional Science and Vitaminology, 2012;58(2):96-104 

16. Kim YJ, Kim SM, Jeong DK, et al. Assocoiations between metabolic syndrome and type of dementia: analysis based on the Naţional Health Insurance Service database of Gangwon province în South Korea. Diabetol Metab Syndr 2021;13(4):1-12.

\section{Correspondence}

Cristina-Maria Gavrilescu,

Lecturer, MD, PhD, „Grigore T. Popa” University of Medicine and Pharmacy, Iași, Ist Medical Department, Iași, Romania, Senior in Internal Medicine, Specialist în Geriatrics, Specialist în Clinical Pharmacology,cristina.gavrilescu@umfiasi.ro

Submission: 30 apr 2021

Acceptance: 03 jun 2021 